\title{
Interconection and Damping Assignment Control of the Subactuated TORA Mechanical System
}

\author{
Maribel Pérez Pirela*1 ${ }^{*}$ Atilio Morillo ${ }^{2}$, Miguel E. Rodriguez R. ${ }^{\dagger 3}$, \\ Dolores Amada Gualli Bonilla ${ }^{\ddagger 4}$, and María Fernanda Romero Villacrés ${ }^{\S 5}$ \\ ${ }^{1}$ Environmental Engineering, Faculty of Engineering, National University of Chimborazo, Riobamba, Ecuador-060150. \\ ${ }^{2}$ Centro de Investigación en Matemática Aplicada, Facultad de Ingeniería Universidad del Zulia, Maracaibo, Venezuela \\ ${ }^{3}$ Career of Pedagogy in Physics and Mathematics, Faculty of Education, National University of Chimborazo, Riobamba, \\ Ecuador-060150. \\ ${ }^{4,5}$ Industrial engineering, Faculty of Engineering, National University of Chimborazo, Riobamba, Ecuador-060150.
}

ORCID: 0000-0002-9687-5471 (Maribel), 0000-0001-8477-6612 (Miguel), 0000-0002-3870-7461 (Dolores), 0000-0003-3122-8131 (Maria)

\begin{abstract}
The TORA system (translational oscillator with rotational actuator) is a benmarch subactuated mechanical system who has attracted great attention of the non-linear control scientific community. In this paper, beginning with the Port Controlled Hamiltonian representation, founded in the total energy of the system considered as kinetic plus potential energy, and based in the use of the interconnection and damping assignment methodology of control, a controller is obtained that achieves the goal of to stabilize global and asymptotically the equilibrium point, with an excellent performance. Numerical simulations at the end of the work confirm the theoretic results.
\end{abstract}

Keywords : Nonlinear control, subactuated mechanical systems, passivity based control, IDA-PBC method, TORA system.

\section{INTRODUCTION}

The control of underactuated systems is an open and interesting problem in mechatronics and robotic systems, both from the theoretical and practical aspects. Although there are several special cases where underactuated systems have been controlled, there are relatively few general principles and exhaustive methods. Simultaneously, the less general problem of stabilizing underactuated nonlinear systems has attracted the attention of the control community for many years [1] and [2].

The design method through interconnection and damping allocation is a method based on the concept of passivity, hence is usually summarized with the initials IDA-PBC, Ortega et al [3]. This method involves nonlinear systems with degree of subactuation one, and through its application the (asymptotic) stabilization of mechanical systems is achieved, at the same time that the Hamiltonian structure is assigned to the closed loop system with respect to a desired energy function [4].

In this work the problem of asymptotic stabilization of the

\footnotetext{
*Orcid:https://orcid.org/0000-0002-9687-5471

† Orcid:https://orcid.org/0000-0001-8477-6612

$\ddagger$ Orcid:https://orcid.org/0000-0002-3870-7461

$\S$ Orcid:https://orcid.org/0000-0003-3122-8131
}

TORA system is considered. This system is one example of these few underactuated systems than can be controlled, and this is achieved by using different approaches, and recently, many nonlinear controller design methods have been applied to stabilize it. Starting from its introduction by [5] as a model for feedback control, first developed examples constitute the approachs of [6], [7] and [8], where cascade control approaches and passivity-based approaches are proposed, with which regulation and stabilization by feedback of the output of the TORA system are obtained.

In our case, using a control law synthesized by applying the interconnection and damping allocation allocation methodology, which is usually summarized with the initials IDA-PBC, through which the aforementioned paradigm is inverted, so the molding of potential energy is first resorted to, and the molding of kinetic energy is performed from the result.

More recently, [9] designed self-tunnig fuzzy sliding mode control using the decoupled method. In addition [10] developed the model by applying the Euler-Lagrange equations, and the design of sliding model control is presented. In, [11], a full constructive procedure is presented, which obvious the necessity of solving PDE to shape the energy for a class of mechanical systems.

In our case, using a control law synthesized by applying the IDA-PBC methodology, through which the aforementioned paradigm is inverted, so the molding of potential energy is first resorted to, and the molding of kinetic energy is performed from the result. This methodology entails the resolution of a set of PDE usually called matching equations. This step remains the stumbling block for the wider application of the different methods associated to the control of systems by using the IDA-PBC approach. However, in the case of the TORA system we have been able to apply a simplification procedure that allowed us to solve satisfactorily that matching equations.

Section 2 summarizes the general aspects of the IDA-PBC Method applied to subactuated mechanical systems developed in [1], Section 3 describes the mathematical model of the TORA system, and Section 4 presents the analysis and design of the controller that manages to stabilize the system. Section 5 presents some numerical simulations that verify the eficiency 
of the designed controller, and finally, Section 6 presents the conclusions of the work.

\section{STABILIZATION OF MECHANICAL SYSTEMS SUBACTUADOS BY IDA-PBC}

\subsection{Realization of the mechanical system in Hamiltonian form controlled by ports}

Consider a sub-actuated mechanical system with total energy expressed as

$$
H(q, p)=\frac{1}{2} p^{T} M^{-1}(q) p+V(q)
$$

where $q \in \mathbb{R}^{n}$ and $p \in \mathbb{R}^{n}$ represent the generalized position and the generalized moment, $M(q)=M^{T}(q)>0$, is the inertia matrix of the system, and $V(q)$ is the potential energy.

$$
\left[\begin{array}{c}
\dot{q} \\
\dot{p}
\end{array}\right]=\left[\begin{array}{cc}
0 & I_{n} \\
-I_{n} & 0
\end{array}\right]\left[\begin{array}{c}
\nabla_{q} H \\
\nabla_{p} H
\end{array}\right]+\left[\begin{array}{c}
0 \\
G(q)
\end{array}\right] u
$$

where $I_{n}$ is the identity matrix $n \times n, \nabla_{q} H$ and $\nabla_{p} H$ are the vectors column gradient of $H$ with respect to $q$ and with respect to $q$, and $u \in \mathbb{R}^{m}$ is the control function.

The matrix $G \in \mathbb{R}^{n \times m}$ is determined by the way the control $u \in \mathbb{R}^{m}$ enters the system, and is invertible in the event that the system is fully operated, that is, $m=n$. This article considers the case in which the system is subactuated, that is, fewer controls than degrees of freedom, and we assume that range $(G)=m$.

In the application of the IDA-PBC method, two basic stages are followed (Ortega et al. [1], Mahindrakar et al. [4]): (1) the stage of the molding of energy, in which the function of total energy of the system, considered as the sum of the kinetic energy plus the potential energy, to assign the desired equilibrium state; and (2) the damping injection stage, which enables asymptotic stability to be achieved. The stabilization mechanism is interpreted in terms of the concept of energy, for which it is necessary to obtain an embodiment of the closed-loop system in the so-called Hamiltonian form controlled by ports (Ortega et al. [3]).

The form of equation 1 motivates proposing the following form for the desired energy of the closed loop system

$$
H(q, p)=\frac{1}{2} p^{T} M_{d}^{-1}(q) p+V_{d}(q)
$$

where $M_{d}=M_{d}^{T}>0$ and $V_{d}$ represent the closed-loop inertia matrix and the desired potential energy function, respectively, and which are to be defined. A basic requirement is that the desired energy $V_{d}$ has an isolated minimum in $q^{*}$, that is

$$
q^{*}=\operatorname{argmin} V_{d}(q)
$$

In passive-based control theory (PBC) the control input is usually broken down into two terms (see Olfati -Know [4])

$$
u=u_{e s}(q, p)+u_{d i}(q, p)
$$

where the first term is designated to achieve energy molding, while damping is introduced into the system through the second term. In this case, the closed-loop dynamics takes the Hamiltonian form controlled by the following ports.

$$
\left[\begin{array}{c}
\dot{q} \\
\dot{p}
\end{array}\right]=\left[J_{d}(q, p)+R_{d}(q, p)\right]\left[\begin{array}{c}
\nabla_{q} H \\
\nabla_{p} H
\end{array}\right]
$$

where the terms

$$
\begin{gathered}
J_{d}=\left[\begin{array}{cc}
0 & M_{d} M^{-1} \\
-M_{d} M^{-1} & J_{2}(q, p)
\end{array}\right] \\
\text { and } R_{d}=\left[\begin{array}{cc}
0 & 0 \\
0 & G K_{v} G^{T}
\end{array}\right] \geq 0
\end{gathered}
$$

they represent the desired interconnection and buffer structures. The antisymmetric matrix $J_{2}$ (and some elements of the matrix $M_{d}$ ) are introduced as free parameters, while the presence of the term $M^{-1} M_{d}$ is justified to preserve the relationship $\dot{q}=M^{-1} p$. On the other hand, as is well known, the contribution of damping in passive systems is achieved via negative feedback of the new passive output, which in this case is given by $G^{T} \nabla_{p} H_{d}$. This is the reason why the expression of equation 5 will choose the expression

$$
u_{d i}=-K_{v} G^{T} \nabla_{p} H_{d}
$$

where $K_{v}=K_{v}^{T}>0$ is taken. This justifies the block $(2,2)$ in the definition of $R_{d}$.

\subsection{Stability}

For the required stability in closed loop the following proposition applies.

Proposition 1. The system 2 with $H_{d}$ given by 3 and $q^{*}$ given by 4 has a stable equilibrium point at. This balance is asymptotically stable if it is locally detectable from the output. Demostration: See [6]

\subsection{Energy molding}

To get the energy molding term $u_{e s}$ in the controller, replace 5 and 7 in 2 and equate the result with 6 , i.e.

$$
\begin{aligned}
& {\left[\begin{array}{cc}
0 & I_{n} \\
-I_{n} & 0
\end{array}\right]\left[\begin{array}{c}
\nabla_{q} H \\
\nabla_{p} H
\end{array}\right]+\left[\begin{array}{l}
0 \\
G
\end{array}\right] u_{e s}} \\
& =\left[\begin{array}{cc}
0 & M_{d} M^{-1} \\
-M_{d} M^{-1} & J_{2}(q, p)
\end{array}\right]\left[\begin{array}{c}
\nabla_{q} H_{d} \\
\nabla_{p} H_{d}
\end{array}\right]
\end{aligned}
$$

where the $R_{d}$ term of 6 has been canceled with the $u_{d i}$ term of 7 . 
The first row of equation 8 produces an identity, while the second row can be expressed as

$$
G u_{e s}=\nabla_{p} H-M_{d} M^{-1} \nabla_{q} H_{d}+J_{2} M_{d}^{-1} p
$$

In the case at hand, the system is subactuated, so $G$ is not invertible, but at most a maximum column range, and therefore the control $u_{e s}$ only influences the terms in the image space of operator $G$. This observation leads to the next set of constraint equations, which must be satisfied for any choice of $u_{e s}$

$$
G^{\perp}\left\{\nabla_{p} H-M_{d} M^{-1} \nabla_{q} H_{d}+J_{2} M_{d}^{-1} p\right\}=0
$$

where $G^{\perp}$ is a left override of maximum range of $G$ (i.e., $\left.G^{\perp} G=0\right)$.

Equation 11 is a set of nonlinear partial differential equations (EDPs) with unknowns $M_{d}$ y $V_{d}$, and with $J_{2}$ being a free parameter, while $p$ it is an independent coordinate. If a solution to this equation can be obtained, the resulting control law $u_{e s}$ would be given by

$$
u_{e s}=\left(G^{\perp} G\right)^{-1} G^{\perp}\left\{\nabla_{p} H-M_{d} M^{-1} \nabla_{q} H_{d}+J_{2} M_{d}^{-1} p\right\}
$$

The partial derivative equations 11 can naturally be separated into terms that depend on $p$ and terms that are independent of $p$, that is, those that correspond to kinetic energy, and those that correspond to potential energy, respectively. In this sense, equation 11 is equivalent to the pair of equations

$$
\begin{gathered}
G^{\perp}\left\{\begin{array}{c}
\nabla_{q}\left(p^{T} M^{-1} p\right)-M_{d} M^{-1} \nabla_{q}\left(p^{T} M_{d}^{-1} p\right) \\
+2 J_{2} M_{d}^{-1} p
\end{array}\right\}=0 \\
G^{\perp}\left\{\nabla_{q} V-M_{d} M_{-1} \nabla_{q} V\right\}=0
\end{gathered}
$$

The first equation is a nonlinear partial differential equation that must be solved for the unknown elements of the closed-loop inertia matrix $M_{d}$. Known this $M_{d}$, equation 14 is a linear partial derivative equation, and therefore easier to solve, so that the greatest difficulty is in solving 13. A simplification, which would considerably reduce the difficulties, is to assume the existence of an inertia matrix $M_{d}$ of constant terms, which would allow us to focus only on finding the desired potential energy $V_{d}$.

\section{MATHEMATICAL MODEL OF THE TORA SYSTEM}

The so-called translational oscillator with rotational actuator (TORA) system was first introduced in Jankovic et al. [7]. Figure 1 illustrates the TORA system consisting of a mass platform $m_{1}$, which can oscillate without friction in the horizontal plane. Located on the platform is an eccentric rotating mass $m_{1}$, it is actuated by a DC motor. Its movement exerts a force on the platform that can be used to dampen translational oscillations. The problem is of interest as a case study in the design of nonlinear controls because the model exhibits a nonlinear interaction between its translational and rotational movements.

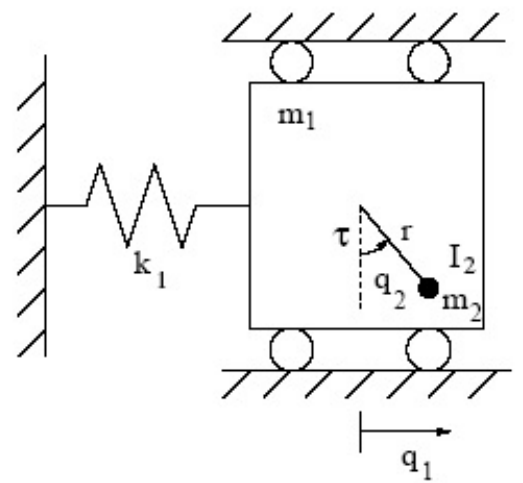

Figure 1: The TORA system

The inertia matrix of the system has the form

$$
M=\left[\begin{array}{cc}
m_{1}+m_{2} & m_{2} r \cos \left(q_{2}\right) \\
m_{2} r \cos \left(q_{2}\right) & m_{2} r^{2}+I
\end{array}\right]
$$

with potential energy given by

$$
V\left(q_{1}, q_{2}\right)=\frac{1}{2} K q_{1}^{2}+m_{2} r g \cos \left(q_{2}\right)
$$

$K$ being the stiffness constant of the spring.

Denoting $c_{1}=m_{1}+m_{2}, c_{2}=m_{2} r, c_{3}=m_{2} r^{2}+I$ and defining the generalized moment of inertia by $p=M \dot{q}$ the inertia matrix $M$ is written as

$$
M\left(q_{2}\right)=\left[\begin{array}{cc}
c_{1} & c_{2} \cos \left(q_{2}\right) \\
c_{2} \cos \left(q_{2}\right) & c_{3}
\end{array}\right]
$$

where, in order to obtain invertible $M$, it must be fulfilled

$$
c_{1} c_{3}-c_{2}^{2}>0
$$

and the total energy function of the system is expressed as

$$
H(q, p)=\frac{1}{2} p^{T} M^{-1}\left(q_{2}\right) p+V\left(q_{1}, q_{2}\right)
$$

Therefore, the Hamilton equations corresponding to the TORA system are given by

$$
\left[\begin{array}{c}
\dot{q} \\
\dot{p}
\end{array}\right]=\left[\begin{array}{cc}
0 & I_{n} \\
-I_{n} & 0
\end{array}\right]\left[\begin{array}{c}
\nabla_{q} H \\
\nabla_{p} H
\end{array}\right]+\left[\begin{array}{c}
0 \\
G
\end{array}\right] u
$$

with $G=\left[\begin{array}{cc}0 & 1\end{array}\right]^{T} \in \mathbb{R}^{2 \times 1}$ and $u \in \mathbb{R}$, thus resulting in a system with 2 degrees of freedom, with degree of subactuation 1 , and with $q_{2}$ as the actuated coordinate.

\section{STABILIZATION OF THE TORA SYSTEM}

\subsection{Energy Shaping in the TORA System}

For the shaping of energy, attention can be focused on the resolution of the EDP 14 . For this, the matrix $M_{d}$ is defined by

$$
M_{d}=\left[\begin{array}{ll}
a_{1} & a_{2} \\
a_{2} & a_{3}
\end{array}\right], \quad a_{1}>0 \quad \mathrm{y} \quad a_{1} a_{3}-a_{2}^{2}>0
$$


Equation 14 for the system is expressed as

$$
\begin{aligned}
& {\left[\frac{a_{1} c_{3}-a_{2} c_{2} \cos \left(q_{2}\right)}{a_{2} c_{1}-a_{1} c_{2} \cos \left(q_{2}\right)}\right] \nabla q_{1} V_{d}+\nabla q_{2} V_{d}} \\
& =\left[\frac{\delta}{a_{2} c_{1}-a_{1} c_{2} \cos \left(q_{2}\right)}\right] K q_{1}
\end{aligned}
$$

Denote

$$
\frac{\gamma_{1}}{\gamma_{2}}=\frac{a_{1} c_{3}-a_{2} c_{2} \cos \left(q_{2}\right)}{a_{2} c_{1}-a_{1} c_{2} \cos \left(q_{2}\right)}=\frac{b_{3}+b_{4} \cos \left(q_{2}\right)}{b_{1}+b_{2} \cos \left(q_{2}\right)}
$$

where $b_{1}=a_{2} c_{1}, b_{2}=-a_{1} c_{2}, b_{3}=a_{1} c_{3}$ and $b_{4}=-a_{2} c_{2}$. So,

$$
\frac{\gamma_{1}}{\gamma_{2}}=\frac{b_{4}}{b_{2}}+\frac{b_{3} b_{2}-b_{1} b_{4}}{b_{2}} \frac{1}{b_{1}+b_{2} \cos \left(q_{2}\right)}
$$

In this way we obtain

$$
\frac{\gamma_{1}}{\gamma_{2}}=\frac{a_{2}}{a_{1}} \Longleftrightarrow a_{2}= \pm \sqrt{\frac{c_{3}}{c_{1}}} a_{1}=\alpha a_{1}
$$

Hereinafter, for simplicity, we will only take the positive value of the constant over the set

$$
\left\{\begin{array}{c}
\left(a_{1}, a_{2}, a_{3}\right) \in \mathbb{R}^{3} / a_{1}, a_{2} \text { y } a_{3} \\
\text { satisfy } 25 \text { and also } a_{2}=\alpha a_{1}
\end{array}\right\}
$$

equation 14 takes the form

$$
\frac{\alpha}{q_{q}} \nabla q_{1} V_{d}+\frac{1}{q_{1}} \nabla q_{2} V_{d}=\frac{K}{a_{1}}\left[\sqrt{c_{1} c_{3}}+c_{2} \cos \left(q_{2}\right)\right]
$$

Applying the characteristics method to equation 27 leads to the solution

$$
\begin{aligned}
V_{d}\left(q_{1}, q_{2}\right) & =F(s)+\frac{K}{a_{1}} \sqrt{c_{1} c_{3}} \frac{1}{2 \alpha}\left[q_{1}^{2}-\left(q_{1}-\alpha q_{2}\right)^{2}\right]+ \\
& \frac{K}{a_{1}} c_{2} \alpha \cos \left(q_{2}\right)+\frac{K}{a_{1}} c_{2} \alpha q_{2} \sin \left(q_{2}\right) \\
& +\frac{K}{a_{1}} c_{2}\left(q_{1}-\alpha q_{2}\right) \sin \left(q_{2}\right)-\frac{K}{a_{1}} c_{2} \alpha
\end{aligned}
$$

where $F(s)$ is an arbitrary function of the variable $s=$ $q_{1}-\alpha q_{2}$. To assign the equilibrium point at the origin to the function $V_{d}$, the function $F(s)$ can be chosen as $F(s)=$ $\frac{1}{2} R\left(q_{1}-\alpha q_{2}\right)^{2}$, which finally results in the desired potential energy

$$
\begin{aligned}
V_{d}\left(q_{1}, q_{2}\right) & =\frac{1}{2} R\left(q_{1}-\alpha q_{2}\right)^{2} \\
& +\frac{K}{a_{1}} \sqrt{c_{1} c_{3}} \frac{1}{2 \alpha}\left[q_{1}^{2}-\left(q_{1}-\alpha q_{2}\right)^{2}\right] \\
& +\frac{K}{a_{1}} c_{2} \alpha \cos \left(q_{2}\right)+\frac{K}{a_{1}} c_{2} \alpha q_{2} \sin \left(q_{2}\right) \\
& +\frac{K}{a_{1}} c_{2}\left(q_{1}-\alpha q_{2}\right) \sin \left(q_{2}\right)-\frac{K}{a_{1}} c_{2} \alpha
\end{aligned}
$$

donde se ha introducido $R$ como un parámetro de diseño. The function $V_{d}\left(q_{1}, q_{2}\right)$ satisfies $V_{d}(0,0)=0$, and the system of equations

$$
\nabla q V_{d}\left(q_{1}, q_{2}\right)=0
$$

own the pair of solutions

$$
\begin{aligned}
& \text { i }\left(q_{1}, q_{2}\right)=\left(0, q_{2}^{*}\right) \quad \text { con } q_{2}^{*} \text { satisfying: } \sin \left(q_{2}\right)= \\
& \frac{a_{1}}{K c_{2}}\left[R \alpha-\frac{K}{a_{1}} \sqrt{c_{1} c_{3}}\right] q_{2}, \mathrm{y} \\
& \text { ii }\left(q_{1}, q_{2}\right)=\left(q_{1}^{* *}, q_{2}^{* *}\right) \operatorname{con} q_{2}^{* *}=\pi-\cos ^{-1}\left(\frac{\sqrt{c_{1} c_{3}}}{c_{2}}\right)
\end{aligned}
$$

Option (ii) is discardable because, according to $21, \frac{\sqrt{c_{1} c_{3}}}{c_{2}}>1$, so that only solution (i) is possible. The relation of (i) is satisfied for at most three values of $q_{2}^{*}$, but it will only have the solution $q_{2}^{*}=0$ if the design parameter $R$ is chosen so that $\frac{a_{1}}{K c_{2}}\left[R \alpha-\frac{K}{a_{1}} \sqrt{c_{1} c_{3}}\right]>1$. In conclusion,

$$
\begin{array}{r}
\nabla q V_{d}\left(q_{1}, q_{2}\right)=0 \Longleftrightarrow\left(q_{1}, q_{2}\right)= \\
(0,0) \text { si } R>\frac{K}{\alpha a_{1}}\left[\sqrt{c_{1} c_{3}}+c_{2}\right]
\end{array}
$$

In such a way that, to have a minimum point for $V_{d}\left(q_{1}, q_{2}\right)$, it only remains to examine what happens with the Hessian of $V_{d}$ at $(0,0)$. For this purpose, the determinant of the Hessian matrix of $V_{d}$ at $(0,0)$ is calculated as

$$
\operatorname{det}\left(\text { Hess } V_{d}(0,0)\right)=\frac{K}{a_{1}^{2}}\left\{\begin{array}{c}
R \alpha a_{1} \sqrt{c_{1} c_{3}} \alpha+R \alpha a_{1} c_{2} \alpha \\
-K c_{1} c_{3}-2 K c_{2} \sqrt{c_{1} c_{3}}-K c_{2}^{2}
\end{array}\right\}
$$

Therefore, to have a minimum at the origin, you must choose

$$
\begin{aligned}
\text { i } & R>0 \mathrm{y} \\
\text { ii } & R>\frac{K}{\alpha a_{1}}\left[\sqrt{c_{1} c_{3}}+c_{2}\right]
\end{aligned}
$$

these conditions exactly match 31 .

\subsection{Stability Analysis}

The stability of the equilibrium point of the TORA system follows from the application of Proposition 1. For asymptotic stability we define the residual set

$$
\Omega=\left\{\begin{array}{c}
\left(q_{1}, q_{2}, p_{1}, p_{2}\right) \\
\in \mathbb{R}^{4} / \dot{H}_{d}\left(q_{1}(t), q_{2}(t), p_{1}(t), p_{2}(t)\right)=0
\end{array}\right\}
$$

By applying a series of observations on this set, it is concluded that $\Omega=\{(0,0,0,0)\}$, and, by the Lasalle Principle of Invariance, the origin is an asymptotically stable equilibrium point.

\subsection{Damping injection}

According to 12 the control $u_{e s}$, and since $J_{2}=0$, is given by

$$
u_{e s}=\left[\begin{array}{ll}
0 & 1
\end{array}\right]\left\{\left[\begin{array}{c}
\nabla q_{1} H \\
\nabla q_{2} H
\end{array}\right]-M_{d} M^{-1}\left[\begin{array}{c}
\nabla q_{1} V_{d} \\
\nabla q_{2} V_{d}
\end{array}\right]\right\}
$$

On the other hand, the expression for the controller $u_{d i}$ term is, according to 8

$$
\begin{aligned}
u_{d i} & =K_{v}\left[\begin{array}{ll}
0 & 1
\end{array}\right]\left[\begin{array}{c}
\nabla p_{1} H_{d} \\
\nabla p_{2} H_{d}
\end{array}\right]= \\
-K_{v}\left[\begin{array}{ll}
0 & 1
\end{array}\right] M_{d}^{-1} p & =-K_{v} \frac{1}{a_{1} a_{3}-a_{2}^{2}}\left[a_{1} p_{2}-a_{2} p_{1}\right]
\end{aligned}
$$


To summarize this section we establish the following

Proposition 2. The TORA system 24, with the parameters $c_{1}$, $c_{2}$ y $c_{3}$ subject to the restriction (21), in closed loop with the IDA-PBC $u=u_{e s}+u_{d i}$ control function, with $u_{e s}$ and $u_{d i}$ given by the expressions 35 and 36, has an equilibrium point at the origin, which is globally asymptotically stable.

\section{NUMERICAL SIMULATIONS}

A typical closed-loop system response to the controller is shown in Figures 2 and 3 . As can be seen, the system displays its response in the form of damped oscillations, and stabilization occurs after 60 seconds.

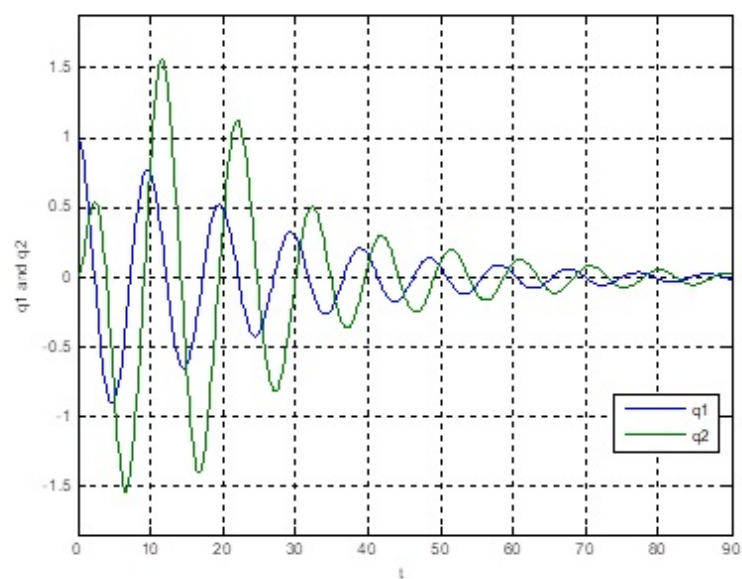

Figure 2: Translational displacement and angular position.

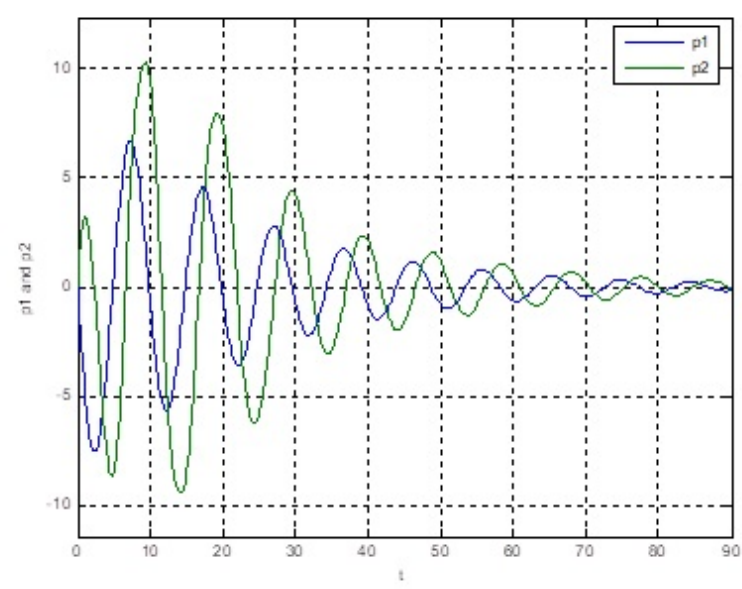

Figure 3: Translational displacement and angular position.

\section{CONCLUSIONS}

In the present work, a control scheme based on IDA-PBC has been developed for the TORA prototype system. The main characteristic of this method is that it exploits the physical structure of the system, in this case, it is exploited that all the information on the dynamic behavior of the mechanical system is contained in the energy and dissipation functions. Consequently, the design of the controller is has concentrated on the management and modification of these variables. For the molding of the total energy, advantage was taken of the possibility of obtaining a desired matrix with constant terms, and for the synthesis of the desired potential energy, a reduction of the set of possible parameters for the desired matrix was obtained, which greatly facilitates the resolution. of the partial differential equation characteristic of the IDA-PBC method. In the end, numerical simulations were performed that show the excellent behavior of the designed controller, significantly reducing the oscillations of the platform, and with a perfectly acceptable "settling time".

\section{REFERENCES}

[1] R.

Ortega,

M. Spong, F. Gómez-Estern, and G. Blankenstein, "Stabilization of a class of underactuated mechanical systems via interconnection and damping assignment," IEEE Transactions on Automatic Control, vol. 47, no. 8, pp. 1218-1233, 2002.

[2] R. Olfati-Saber, "Nonlinear Control of underactuated mechanical systems with applications to robotics and aerospace vehicles," Ph.D. dissertation, Massachussets Institute of Technology, 2001.

[3] R. Ortega, A. van der Schaft, B. Maschke, and G. Escobar, "Interconnection and damping assignment passivity-based control of port-controlled hamiltonian systems," Automatica, vol. 38, no. 4, pp. 585 - 596, 2002.

[4] A. D. Mahindrakar, A. Astolfi, R. Ortega, and G. Viola, "Further constructive results on interconnection and damping assignment control of mechanical systems: the acrobot example," in 2006 American Control Conference, 2006, pp. 6 pp.-.

[5] Chih-Jian Wan, D. S. Bernstein, and V. T. Coppola, "Global stabilization of the oscillating eccentric rotor," in Proceedings of 1994 33rd IEEE Conference on Decision and Control, vol. 4, 1994, pp. 4024-4029 vol.4.

[6] G.

Escobar,

R. Ortega, and H. Sira-Ramírez, "Output-feedback global stabilization of a nonlinear benchmark system using a saturated passivity-based controller," IEEE Transactions on Control Systems Technology, vol. 7, no. 2, pp. 289-293, 1999.

[7] M. Jankovic, D. Fontaine, and P. Kokotović, "Tora example: Cascade- and passivity-based control designs," IEEE Transactions on Control Systems Technology, vol. 4, no. 3, pp. 292-297, 1996.

[8] A. Pavlov, B. Janssen, N. van de Wouw, and H. Nijmeijer, "Experimental output regulation for the tora system," in Proceedings of the 44th IEEE 
International Journal of Engineering Research and Technology. ISSN 0974-3154, Volume 13, Number 10 (2020), pp. 2706-2711 (C)International Research Publication House. https://dx.doi.org/10.37624/IJERT/13.10.2020.2706-2711

Conference on Decision and Control, 2005, pp. 1108-1113.

[9] L.-C. Hung, H.-P. Lin, and H.-Y. Chung, "Design of self-tuning fuzzy sliding mode control for tora system," Expert Systems with Applications, vol. 32, no. 1, pp. 201 - 212, 2007.

[10] H. Ali and M. Kadhim, "A combined h2/sliding mode controller design for a tora system," Al-Nahrain Journal for Engineering Sciences, vol. 21, no. 4, pp. 501-507, Dec. 2018.

[11] P. Arpenti, D. Serra, F. Ruggiero, and V. Lippiello, "Control of the tora system through the ida-pbc without explicit solution of matching equations," in 2019 Third IEEE International Conference on Robotic Computing (IRC), 2019, pp. 381-385. 